\title{
Modeling and Simulation of Big Bus Equipped with Autonomous
}

\section{Emergency Braking System}

\author{
JIANG Wuhua ${ }^{1, a}$, LIU Bolin ${ }^{1, b}$ \\ ${ }^{1}$ School of Automobile and Traffic Engineering, Hefei University of Technology, Hefei 230009, China \\ awhilang@hfut.edu.cn, ${ }^{\mathrm{b}} 930872832 @ q q . c o m$
}

Keywords: Autonomous emergency braking system, active safety, single neuron PID control

\begin{abstract}
Autonomous emergency braking system (AEBS) is important active safety technology, which aims to avoid collision or reduce collisions by automatic braking, has become a hot research topic in recent years. However, Modeling and simulation research on big bus is relatively limited, not systematic introduction. Three typical braking scenarios are defined and the safety situations are assessed by comparing the current distance between the host vehicle and the leading vehicle with the critical braking distance. To reflect the nonlinear time-varying characteristics and control effect of the longitudinal dynamics, the vehicle longitudinal dynamics model is established in TruckSim. Then the braking controller with the structure of upper and lower layers is designed based on the single neuron PID control when confronting emergency braking conditions. Co-simulations utilizing TruckSim and Simulink are finally carried out on a TruckSim vehicle model to explore the effectiveness of the proposed controller of AEBS. Results display that the designed controller has a good response in preventing colliding with the leading vehicle.
\end{abstract}

\section{Introduction}

The autonomous emergency braking system is considered to be at this stage can prevent collision damage because of the driver negligence or the heading vehicle's abnormal emergency braking, which is one of the most effective function of system. Considering the lack of real traffic data in China, there is unable to establish a compatible with the special traffic condition AEB system of collision avoidance strategy. So this paper adopts the safe distance model of longitudinal active collision avoidance system to validate the performance of AEBS. Considering the strong nonlinearity of the vehicle system and the uncertainty factors in the process of driving, the research on modeling and the design of the vehicle dynamic control has been conducted worldwide. For example, Ferrara and Vecchio [1], who were motivated by the robustness features of the sliding mode, designed a second-order sliding mode control system based on a nonlinear vehicle model. Nouveliere and Mammar [2] proposed an experimental implementation of a shared vehicle longitudinal controller based on a second-order sliding mode technique, which was tested under usual traffic conditions such as stop-and-go, stopping at obstacles, and car-following in a number of scenarios, but it mainly concentrated on the low velocity. Lee and Kim [3] controlled the relative velocity and distance of the vehicles by taking advantage of fuzzy logic theory. However, they ignored the influence of the dynamic characteristics of an engine model and the slip characteristics of the tire model, which is difficult to reflect the control effect in the real working conditions. Compared with the fuzzy logic control and the sliding mode control, the single neuron PID control has more strong robustness and can adapt to environmental changes. 


\section{Methodology}

2.1. Safety Distance Model. The autonomous emergency braking system (AEBS) includes the following key techniques: environment perception and processing, evaluation of the traffic safety state, vehicle dynamics modeling, and control execution techniques.

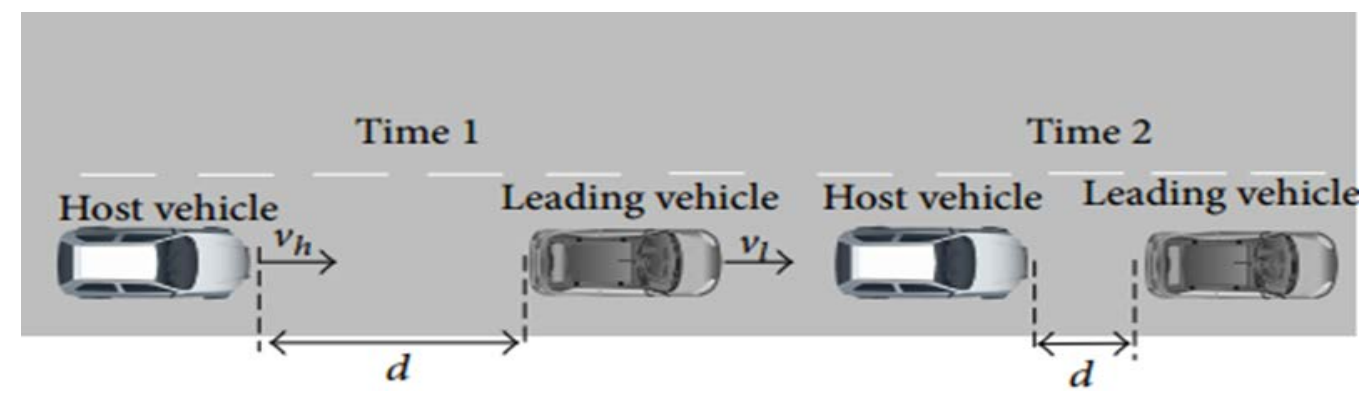

Fig.1: The host vehicle and the leading vehicle's relative position after the host vehicle braking (a) The Leading Vehicle Static. Fig.1 shows a schematic view. $d$ represents the real-time distance between the host vehicle and the leading vehicle. The velocity of the leading vehicle is zero. The critical braking distance can be obtained according to the safety distance model during the braking process as follows [4]:

$$
d_{b r 1}=v_{h}\left(t_{r}+\frac{t_{i}}{2}\right)+\frac{v_{h}^{2}}{2 \mu g}+d_{\text {min }}
$$

where $d_{b r 1}$ is the critical braking distance for decelerating, represents the original velocity of the host vehicle, $t_{r}$ is the sum of response time of the driver and braking coordination time ranging from 0.8 to $1.2 \mathrm{~s}, t_{i}$ is the growth time of the braking deceleration which varies from 0.1 to $0.2 \mathrm{~s}, \mu$ denotes the friction coefficient of the road, $g$ is referred to as the acceleration of gravity, $d_{\min }$ is the minimum distance between the host and the leading vehicle when they stop, ranging from 2 to 5 $\mathrm{m}$. Because the conservative safety distance model is adopted in this article, we should consider that $t_{r}$ is $1.2 \mathrm{~s}, t_{i}$ is $0.2 \mathrm{~s}$, and $d_{\min }$ is $5 \mathrm{~m}$.

(b) The Leading Vehicle Moving at a Constant Speed. The case is similar to the case (a), and the leading vehicle moves at a constant speed. Then the critical braking distance between the host vehicle and the leading vehicle could be calculated by

$$
d_{\text {br } 2}=\left(v_{h}-v_{l}\right)\left(t_{r}+\frac{t_{i}}{2}\right)+\frac{\left(v_{h}-v_{l}\right)^{2}}{2 \mu g}+d_{\text {min }},
$$

where $d_{b r 2}$ is the critical braking distance for decelerating, $v_{h}$ and $v_{l}$ represent the original velocity of the host and the leading vehicle, respectively.

(c) The Leading Vehicle Braking Urgently. If the host vehicle detects the leading vehicle decelerating, the host vehicle must decelerate or even brake to ensure a safety distance between each other. The critical braking distance can be calculated by

$$
d_{b r 3}=v_{h} t_{r}+\frac{\left(v_{h}-v_{l}\right) t_{i}}{2}+\frac{v_{h}^{2}-v_{l}^{2}}{2 \mu g}+d_{\text {min }}
$$

where $d_{b r 3}$ is the critical braking distance for decelerating.

\subsection{Vehicle Model}

(a) The Vehicle Longitudinal Dynamics Model. The vehicle dynamics simulation model can be built utilizing the TruckSim software according to the structural parameters, as well as the model of the engine, transmission, steering, and other components. However, some parts cannot be defined in TruckSim specifically, such as the characteristics of the brake system. For the reason that TruckSim is able to interface with external MATLAB and Simulink easily, those parts can be defined in Simulink; therefore, the vehicle inverse longitudinal dynamics model should be built firstly. 
(b) The Vehicle Inverse Longitudinal Dynamics Model. Considering the situation that controlling the throttle and brake at the same time can lead to system shock in the actual driving process, it is needed to control the throttle and brake according to the desired acceleration [5].

We obtain the maximum acceleration $a_{\max }$ under different velocity with TruckSim by setting the value of throttle to be zero. In order to improve passenger's comfort and to prevent frequent switching between the throttle control and braking control during the controlling process, a buffer zone for the deceleration value is set [6]. Then the switching logic can be expressed as follows:

$a_{\text {des }}>a_{\text {max }}+\Delta a, \quad$ throttle control

$a_{\text {des }} \leq a_{\text {max }}+\Delta a, \quad$ braking control ,

where $a_{d e s}$ is the vehicle desired acceleration, $a_{\max }$ is the maximum acceleration, and $\Delta a$ is the buffer value, which is set to $0.1 \mathrm{~m} / \mathrm{s}^{2}$.

If the judging outcome is the throttle control, the desired engine torque can be calculated according to the requirement of the desired acceleration. Then the throttle aperture can be deduced from the inverse engine model. Ignoring the quality conversion of vehicles rotating parts, the vehicle longitudinal dynamics equation is as follows:

$$
\mathrm{m} a_{d e s}=F_{t}-F_{x b}-\sum F(v) \text {, }
$$

where $\mathrm{m}$ is the whole vehicle mass and $F_{t}$ is the driving force of the vehicle from the road due to the driving effect of the engine, whose value is zero when braking. $F_{x b}$ is the braking force on the vehicle from the road due to the effect of brake, whose value is zero when driving. $\sum F(v)$ is the sum of resistance because of wind and rolling; namely,

$$
\sum F(v)=\frac{1}{2} \mathrm{C}_{\mathrm{D}} A \rho v^{2}+m g f,
$$

where $\rho$ denotes the air density, $C_{D}$ represents the aerodynamic drag coefficient, $A$ indicates the windward area, $v$ is the vehicle velocity, and $f$ denotes the rolling friction coefficient.

Ignoring the elastic deformation of the drive train, the vehicle driving force can be calculated by

$$
F_{t}=\frac{\eta T_{e} \tau\left(\omega_{t} / \omega_{e}\right) R_{g} R_{m}}{r},
$$

where $\tau\left(\omega_{t} / \omega_{e}\right)$ is the torque characteristic curve of the hydraulic torque converter, $T_{e}$ is engine output torque, $R_{g}$ is the transmission gear ratio, $R_{m}$ means the main gearbox reduction ratio, $\omega_{t}$ means the turbine velocity of the hydraulic torque converter, $\omega_{e}$ is the engine velocity, $\eta$ denotes the mechanical efficiency, and $r$ is the tire rolling radius.

$K_{d}$ is a variable and is defined as

$$
K_{d}=\frac{\eta \tau\left(\omega_{t} / \omega_{e}\right) R_{g} R_{m}}{r} \text {. }
$$

The braking force $F x b$ is zero when driving; then, by formulas (5), (6), (7), and (8), the desired engine output torque is obtained as follows:

$$
T_{e}=\frac{F_{t}}{K_{d}}=\frac{m a_{d e s}+(1 / 2) \mathrm{C}_{D} A \rho v^{2}+m g f}{K_{d}} .
$$

When acquiring the desired engine output torque $T_{e}$, the engine velocity $\omega_{e}$, and the reverse engine torque characteristic curve $f\left(T_{e}, \omega_{e}\right)$, the desired throttle opening $\gamma_{\text {des }}$ can be obtained accurately:

$$
\gamma_{\text {des }}=f\left(T_{e}, \omega_{e}\right)
$$

If the judgment result is the brake control, the desired braking torque can be calculated according to the requirements of the desired acceleration. Under the maximum braking force of the road, the braking force can be approximately expressed as a linear function of the oil pressure in the braking 
line, and its expression is as follows:

$$
\frac{T_{b f}+T_{b r}}{r P}=\frac{F_{b}}{P}=K_{b}
$$

where $T_{b f}$ and $T_{b r}$ represent the braking torque of the front and rear wheels, respectively, $r$ is the tire rolling radius, $F_{b}$ expresses the braking force, and $K_{b}$ is the ratio of the brake force and brake pressure.

The driving force $F_{t}$ is zero when braking, so the desired brake pressure $P_{\text {des }}$ can be calculated as

$$
P_{\text {des }}=\frac{\left|m a_{d e s}+(1 / 2) \mathrm{C}_{\mathrm{D}} \mathrm{A} \rho v^{2}+m g f\right|}{K_{b}} .
$$

2.3. Controller Design. The design of control system is the key of autonomous emergency braking system, which aims to realize its function by the control of the vehicle longitudinal dynamics. Considering the big bus capacity is big, in order to ensure the driving safety, this article chooses $-5 \mathrm{~m} / \mathrm{s}^{2}$ as the maximum braking deceleration of the vehicle.

The emergency braking controller employs a hierarchical control structure. In the upper controller, we obtain the expectations of the acceleration by the switch module to choose the largest braking deceleration as the desired acceleration. In the lower controller, the desired acceleration $a_{\max }$ is obtained by the upper controller and then takes the difference between the value of the desired acceleration $a_{\max }$ and the current actual acceleration $a$ as the input of the single neuron PID controller.

The state variables of $\mathrm{x}_{1}, \mathrm{x}_{2}$, and $\mathrm{x}_{3}$ required in the single neuron controller are as follows:

$$
\begin{aligned}
& x_{1}(t)=e(t)=a_{\max }(t)-a(t) \\
& x_{2}(t)=\Delta e(t)=e(t)-e(t-1) \\
& x_{3}(t)=e(t)-2 e(t-1)+e(t-2)=e(t-1)-e(t-2) .
\end{aligned}
$$

Control algorithms and learning rules are shown in the following equation:

$$
\begin{aligned}
& a_{c o n}(t)=a_{c o n}(t-1)+K \sum_{i=1}^{3} \omega_{i}^{\prime}(t) x_{i}(t) \\
& \omega_{i}^{\prime}(t)=\frac{\omega_{i}(t)}{\sum_{i=1}^{3}\left|\omega_{i}(t)\right|} \\
& \omega_{1}(t)=\omega_{1}(t-1)+\mu_{I} e(t) a_{c o n}(t-1)(e(t)+\Delta e(t)) \\
& \omega_{2}(t)=\omega_{2}(t-1)+\mu_{P} e(t) a_{c o n}(t-1)(e(t)+\Delta e(t)) \\
& \omega_{3}(t)=\omega_{3}(t-1)+\mu_{D} e(t) a_{c o n}(t-1)(e(t)+\Delta e(t)),
\end{aligned}
$$

where $\mu_{I}, \mu_{P}$, and $\mu_{D}$ signify the learning rates of integral, proportional, and differential components, respectively. $K$ denotes the proportion coefficient of neurons such that $K>0, \omega_{i}(t)$ corresponds to the weighting coefficient of $x_{i}(t), a_{\max }(t)$ is the desired acceleration, $a(t)$ is the actual acceleration of the host vehicle, and $a_{c o n}(t)$ is the controlled acceleration. 


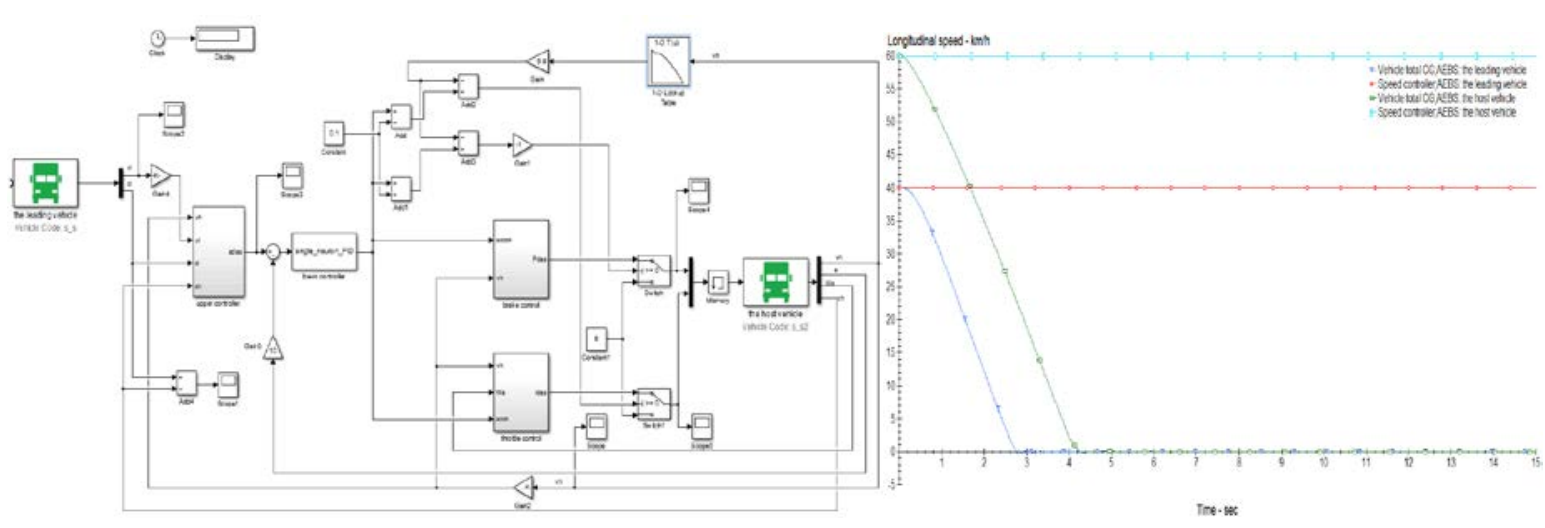

Fig.2: The co-smulation model of TruckSim and Simulink

Fig.3: The vehicle velocity

\section{Simulation}

To test and verify the effect of the controller of AEBS, three typical working conditions are presented for co-simulation utilizing TruckSim and Matlab/Simulink. This paper chooses the main parameters of the big bus prototype of AnHui AnKai Motor Company to build models using TruckSim. The co-smulation model of TruckSim and Simulink is shown in Fig.2.The parameters of the big bus prototype are shown in Table 1. The parameters of the designed controller for emergency are shown in Table 2.

Table 1: AnHui AnKai big bus vehicle parameters

\begin{tabular}{|c|c|c|}
\hline Symbol & Designation & Value \\
\hline$m$ & whole vehicle mass & $13100(\mathrm{~kg})$ \\
\hline$I_{z}$ & moment of inertia around the $\mathrm{z}$ axis & $56724.8\left(\mathrm{~kg} \cdot \mathrm{m}^{2}\right)$ \\
\hline $\mathrm{a}$ & distance from front axle to vehicle mass center & $3881(\mathrm{~mm})$ \\
\hline$b$ & distance from the rear axle to the mass center & $2199(\mathrm{~mm})$ \\
\hline$\rho$ & air density & $1.206\left(\mathrm{~kg} / \mathrm{m}^{3}\right)$ \\
\hline$f$ & rolling friction coefficient & 0.02 \\
\hline$r$ & wheel rolling radius & $487(\mathrm{~mm})$ \\
\hline$H_{g}$ & height of the mass center of the vehicle & $1020(\mathrm{~mm})$ \\
\hline$C_{D}$ & aerodynamic drag coefficient & 0.38 \\
\hline A & windward area & 2.85 \\
\hline$R_{m}$ & the main reducer reduction ratio & $\left.\mathrm{m}^{2}\right)$ \\
\hline
\end{tabular}

Table 2: Control parameters for emergency brake

\begin{tabular}{|c|c|c|c|c|}
\hline Symbol & $\mu_{I}$ & $\mu_{P}$ & $\mu_{D}$ & $K$ \\
\hline Value & 20 & 500 & 500 & 0.3 \\
\hline
\end{tabular}

This paper chooses the case (c) as example. In this case, the initial velocity and the braking deceleration of the leading vehicle are $40 \mathrm{~km} / \mathrm{h}$ and $-5 \mathrm{~m} / \mathrm{s}^{2}$, respectively, its initial position is placed $26 \mathrm{~m}$ ahead of the original point. The host vehicle starts to move from the original point with a constant velocity of $60 \mathrm{~km} / \mathrm{h}$. The simulation duration is set to $15 \mathrm{~s}$ in TruckSim. The simulation results are shown in Fig.3 to 5.

As shown in Fig.3, the velocity of the host vehicle drops with maximum braking deceleration, from the initial velocity of $60 \mathrm{~km} / \mathrm{h}$ to zero at $4.2 \mathrm{~s}$ and the velocity of the leading vehicle drops with $-5 \mathrm{~m} / \mathrm{s}^{2}$ of the braking deceleration, from the initial velocity of $40 \mathrm{~km} / \mathrm{h}$ to zero at $2.8 \mathrm{~s}$. Fig. 4 shows that the actual maximum braking deceleration of the host vehicle is nearly accordant with the desired braking deceleration, and the actual braking deceleration gradually reaches the 
maximum braking deceleration $-5 \mathrm{~m} / \mathrm{s}^{2}$ at $3.8 \mathrm{~s}$, then stop to brake. The curve of the leading vehicle has larger fluctuation after braking because of the lack of control. From Fig.5, we could know that the relative distance of the host vehicle and the leading vehicle was $26 \mathrm{~m}$ at the initial time and gradually tends to the desired relative distance. Finally, the relative distance reached $4.9 \mathrm{~m}$ when the host vehicle stops completely, and it close to the minimum distance of safety distance model.

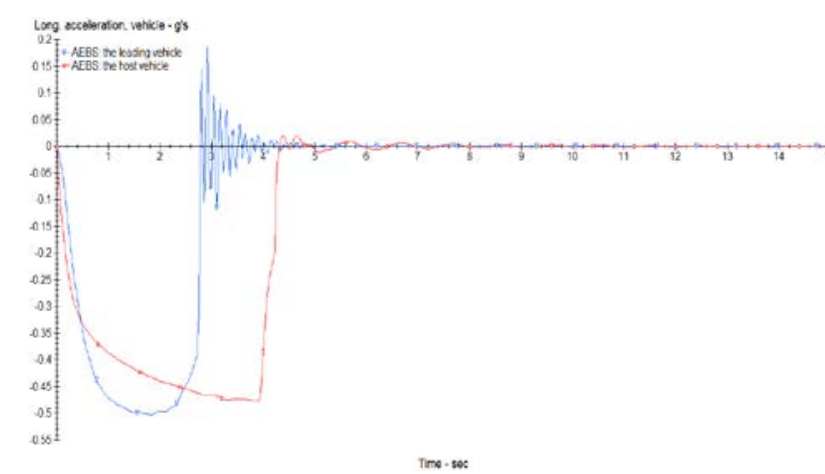

Fig.4: The vehicle deceleration

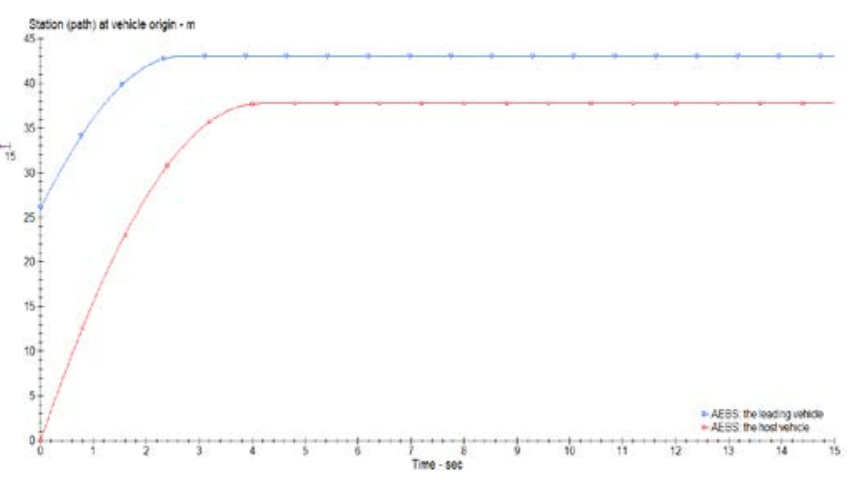

Fig.5: The relative distance

\section{Conclusion}

According to the requirement of the autonomous emergency braking system (AEBS), the safety status was judged by comparing the current distance between the host vehicle and the leading vehicle with the critical braking distance. Then the vehicle dynamics model and the vehicle inverse longitudinal dynamics model were established in TruckSim. The collision avoidance controller was designed based on single neuron PID method, realizing automatic braking control of the host vehicle for vehicle collision avoidance and guaranteeing the active safety of the vehicle. The parameters of the experimental vehicle were applied to the vehicle model utilizing TruckSim, which could better reflect nonlinear time-varying characteristics and control effect of the longitudinal dynamics, possessing a certain practical significance and research value. Finally, co-simulations were carried out on a TruckSim vehicle model utilizing Matlab/Simulink to explore the effectiveness of the proposed controller of AEBS. Results indicate that the controller can realize the emergency brake when there is a vehicle ahead of the host vehicle.

\section{References}

[1] A. Ferrara and C. Vecchio, "Second-order sliding mode control of a platoon of vehicles," International Journal of Modelling, Identification and Control, vol. 3, no. 3, pp. 277-285, 2008.

[2] L. Nouveliere and S. Mammar, "Experimental vehicle longitudinal control using a second order sliding mode technique,” Control Engineering Practice, vol. 15, no. 8, pp. 943-954, 2007.

[3] G. D. Lee and S. W. Kim, "A longitudinal control system for a platoon of vehicles using a fuzzy-sliding mode algorithm,” Mechatronics, vol. 12, no. 1, pp. 97-118, 2002.

[4] D. Y. Qu, X. F. Chen, W. S. Yang, and X. H. Bian, "Modeling of car-following required safe distance based on molecular dynamics," Mathematical Problems in Engineering, vol. 2014, Article ID 604023, 7 pages, 2014.

[5] M. H. Lee, H. G. Park, S. H. Lee, K. S. Yoon, and K. S. Lee, “An adaptive cruise control system for autonomous vehicles,” International Journal of Precision Engineering and Manufacturing, vol. 14, no. 3, pp. 373-380, 2013.

[6] P. Li, M. X. Wei, and X. L. Hou, "Modeling and co-simulation of adaptive cruise control system,” Automotive Engineering, vol. 34, no. 7, pp. 622-626, 2012. 